\title{
EDITORIAL \\ CARACTERIZACIÓN DE LAS REVISTAS \\ CIENTÍFICO-ACADÉMICAS DE EDUCACIÓN \\ FÍSICA Y ÁREAS AFINES EN COLOMBIA, \\ AÑO 2018-1
}

\section{CARACTERIZAÇÃO DAS REVISTAS CIENTÍFICO ACADÊMICAS DA ẺDUCAÇÃO FÍSICA E ÁREAS AFINS DA COLÔMBIA NO ANO 2018-1}

CHARACTERIZATION OF THE SCIENTIFIC-ACADEMIC JOURNALS OF PHYSICAL EDUCATION AND RELATED AREAS IN COLOMBIA, YEAR 2018-1

\section{Elí Adonay Castaño Ospina'}

1 Licenciado en Educación Física y Deporte de la Universidad de Antioquia.

E-mail: adonay.castano@gmail.com

Castaño Ospina, E. A. (2019). Caracterización de las Revistas Científico-Académicas de Educación Física y Áreas afines en Colombia, año 2018-1. Educación Física y Deporte, 38(2), 217-238. Jul.-Dic. http://doi.org/10.17533/udea.efyd.v38n2a01

\section{RESUMEN}

Objetivo: caracterizar las revistas científico-académicas de educación física y áreas afines en Colombia, para identificar en qué estado se encuentra su nivel de desarrollo y los diferentes aspectos editoriales. Método: investi- 
gación de tipo descriptivo, en la que se aplicó una matriz de evaluación a seis revistas del campo, en el semestre 1 de 2018. Resultados: se describe el estado de las revistas seleccionadas en dimensiones como: calidad formal, informativa, editorial y calidad científica; difusión y visibilidad. Conclusión: se sugiere emprender acciones de mejora con base en los hallazgos planteados en la presente evaluación.

PALABRAS CLAVE: educación física, publicación, revistas, calidad, evaluación.

\section{ABSTRACT}

Objective: To characterize the scientific-academic journals in physical education and related areas in Colombia, to identify the state of their level of development and the different editorial aspects. Method: Descriptive research, in which an evaluation matrix was applied to six physical education journals and related areas of the field, in semester 1 of 2018. Results: The status of the selected journals is described in dimensions such as formal, informative, editorial, and scientific quality; diffusion and visibility. Conclusion: It is suggested to undertake improvement actions based on the findings of this evaluation.

KEYWORDS: physical education; publishing; journals, quality; evaluation.

\section{RESUMO}

Objetivo: caracterizar as revistas científico - acadêmicas em educação física e áreas afins em Colômbia com o intuito de identificar seu nível de desenvolvimento e seus diferentes aspetos editoriais. Método: pesquisa de tipo descritiva, uma matriz de avaliação foi aplicada a seis revistas de educação física e áreas afins no primeiro semestre de 2018. Resultados: descreve-se o estado das revistas eleitas em suas múltiplas dimensões, tais como: qualidade formal, qualidade informativa, qualidade editorial, qualidade científica, divulgação e visibilidade. Conclusão: Recomenda-se realizar ações para melhorar as revistas baseados nos resultados propostos a partir dessa avaliação.

PALAVRAS-CHAVES: educação física; publicação; qualidade; revistas; avaliação. 


\section{INTRODUCCIÓN}

Las revistas son un medio para la comunicación de la investigación académica y científica que se produce en las diferentes ciencias y campos de conocimiento, por lo que deben ofrecer amplia visibilidad a la comunidad a la cual se dirigen. Como lo expresan Osca et al. (2008), son "el vehículo convencional para hacer públicos los descubrimientos, los nuevos métodos e incluso las nuevas hipótesis". El contenido de las revistas permite divulgar conocimientos que aportan, por su novedad y con su aplicación, al desarrollo y mejoramiento (Day, 2005, p.1). Cualificar las revistas del campo de la Educación Física en Colombia posibilitaría optimizar la difusión del conocimiento.

En el estudio de Rozemblum et al. (2015) se analizaron los parámetros de evaluación establecidos por las bases de datos RedALyC, Latindex, SciELO, Scopus y Web of Science para incorporar revistas científicas en sus colecciones, y concluyen que el aporte de las bases de datos es importante para cumplir criterios básicos de calidad editorial, destacando el apartado de visibilidad, pues para Scopus el $47 \%$ de parámetros evaluados a las revistas se refiere a visibilidad, y el $60 \%$ de indicadores de calidad valorados por RedALyC se ocupan de este aspecto (Rozemblum et al., 2015, p.76). También concluyen que, para "medir la calidad científica de las revistas [...] resulta necesario definir otros criterios más allá de que estén o no incluidas en ciertos sistemas de indización basados en evaluación de 'calidad editorial' (Rozemblum et al., 2015 p.77).

Rodríguez et al. (2007) se ocupan de la métrica, revisando la producción científica de las revistas de Ciencias Sociales de Cuba, destacando las revistas más productivas, las más prolíficas, la escasa colaboración entre los centros docentes y de investigación, y los problemas relacionados con la calidad de las publicaciones.

Molina et al. (2016) evaluaron 35 revistas publicadas en la Universidad de Antioquia (Colombia), aplicando criterios de inclusión de bases de datos como Latindex, Publindex y el Manual 
de buenas prácticas en la edición de revistas científicas, de la Fundación de Ciencia y Tecnología de España (FECYT). El estudio permitió conocer el estado de las revistas, su calidad formal, informativa, científica, de gestión administrativa y su nivel de difusión.

En cuanto a la gestión, analizan la composición y dedicación del equipo administrativo de la revista (director, editor, asistente editorial, editores de sección), mencionando el tiempo dedicado según el cargo. En el análisis se observa la ausencia de varios cargos en las revistas; se aprecian revistas con un solo cargo (editor con 27 horas a la semana) responsable de la edición, otras con dos cargos (editor y asistente) y algunas con cuatro cargos, pero ninguno tiene en su totalidad los cargos que, en teoría, debería componer un equipo de trabajo adecuado para las revistas, según el manual FECYT (Molina et al., 2016 p.64).

Teniendo como base los estudios mencionados, surge el interés investigativo por las revistas del campo de la Educación Física en Colombia, debido al escaso conocimiento al respecto.

\section{MÉTODO}

La presente investigación es de tipo descriptivo. Dado que el mayor esfuerzo en el proceso editorial de los artículos científico - académicos es de las universidades, con una participación del 61\% (Corera \& Molina, 2016), se seleccionaron para análisis 6 revistas pertenecientes a universidades colombianas, encargadas de publicar artículos resultados de investigación en el área de educación física y afines, en 2018. A estas revistas se les aplicó una matriz desarrollada por Molina et al. (2016), quienes la implementaron, como se mencionó, para analizar revistas de la Universidad de Antioquia, identificando aspectos a mejorar en su gestión. Con autorización de los investigadores, esta matriz se adaptó a las dimensiones y propósitos del presente estudio, en los siguientes aspectos: 
- Dimensión Administrativa

- Calidad Formal

- Calidad Informativa

- Calidad Editorial

- Calidad Científica

- Difusión y Visibilidad

En el presente trabajo se busca conocer el estado de las revistas en diferentes aspectos fundamentales relacionados con su adecuada gestión. La evaluación se realizó a los números publicados entre enero de 2016 y enero de 2018. Las revistas se seleccionaron por medio de los siguientes criterios:

- Estar incluidas en la base de datos nacional Publindex?

- Pertenecer al área de Educación Física.

- Tener un carácter científico - académico.

- Estar activas en el año 2016.

- Ser de acceso libre (Open Access).

Las revistas incluidas en el estudio se presentan en la siguiente tabla.

Tabla 1. Revistas universitarias de Colombia en Educación Física. Elaboración propia

\begin{tabular}{ll}
\hline \multicolumn{1}{c}{ Título de la revista } & \multicolumn{1}{c}{ Universidad } \\
\hline Lúdica Pedagógica & Universidad Pedagógica Nacional (Bogotá) \\
\hline Ímpetus & Universidad de los Llanos (Villavicencio) \\
\hline Educación Física y Deporte & Universidad de Antioquia (Medellín) \\
\hline $\begin{array}{l}\text { Actividad Física y Desarrollo } \\
\text { Humano }\end{array}$ & Universidad de Pamplona (Pamplona) \\
\hline
\end{tabular}

1 Índice Bibliográfico Nacional, conformado por las Revistas Colombianas Especializadas en Ciencia, Tecnología e Innovación -Ctel, clasificadas en las categorías A1, A2, B y C. según criterios de evaluación internacional para publicaciones científicas relacionados con gestión editorial, evaluación, visibilidad e impacto. 
Edu-Física ciencias aplicadas al deporte

Universidad del Tolima (Ibagué

Actividad Física y Deporte

Universidad de Ciencias Aplicadas y

Ambientales (Bogotá)

Con excepción de la Universidad de Ciencias Aplicadas y Ambientales (Bogotá), las demás son universidades públicas. A continuación, se describen las dimensiones evaluadas.

\section{Calidad formal (electrónica)}

Hace referencia al grado de observancia de las normas nacionales e internacionales de publicación científica (Molina et al, 2016, p.31). Para la evaluación de la calidad editorial, se toma en cuenta la calidad de los artículos, las palabras clave y los resúmenes, así como la filiación de los autores y la calidad del sitio Web. Con ello se pretende evaluar la legibilidad de los artículos; que su estructura sea de esquema Introducción, Material y Método, Resultados y Discusión; conocer la nacionalidad y filiación de los autores para evitar la endogamia; y conocer la información del sitio Web, el equipo científico y editorial de las revistas, el empleo de tesauros y los idiomas que se manejan.

\section{Calidad informativa}

Es la encargada de comunicar a los autores sobre las políticas de aceptación y publicación de los artículos, de los procedimientos de revisión, aceptación o rechazo de las publicaciones enviadas, consideraciones éticas y derechos del autor. Da claridad sobre las instrucciones para publicar, evaluar y las políticas de acceso abierto.

\section{Calidad editorial}

En esta se evalúa el proceso editorial, la estructura de la revista en sus órganos de control y evaluación, la manera en la que se 
distribuye el conocimiento. De acuerdo con Molina et al. "su calidad en el proceso editorial está determinada por parámetros como la periodicidad, regularidad, arbitraje científico, revisores, anonimato en la revisión, instrucciones para la revisión, comunicación motivada de las decisiones, consejos de redacción y asesor" (2016).

\section{Calidad científica}

Es una de las dimensiones más importantes. Es la encargada de evaluar el porcentaje de artículos de investigación, que los autores no pertenezcan a la propia institución y que los árbitros y los comités científico y editorial sean libres de endogamia. Analiza la geografía de estos, tanto a nivel nacional como internacional.

\section{Difusión y visibilidad}

Molina et al. señalan que "la difusión de una revista debe entenderse como la capacidad que ésta tiene de ser visible para la comunidad científica a la que se dirige" (2016, p.42). La difusión y la visibilidad son características de las revistas que permiten alcanzar un reconocimiento en la sociedad de científicos o profesionales afines al área objeto de interés. Se requiere que las revistas se encuentren indexadas en bases de datos nacionales e internacionales, para que se logren dar a conocer en diferentes lugares del mundo.

Para las revistas seleccionadas, la evaluación se llevó a cabo en las dimensiones de calidad (formal, informativa, editorial y científica), difusión y visibilidad.

\section{RESULTADOS}

Tras el análisis, se evidenció que las revistas incluidas en el estudio no cumplen con la mayoría de los estándares de una revista científica. La calidad informativa es una de las dimensiones que 
precisa intervención por parte de los encargados de las revistas; las políticas de acceso, el código de ética y las instrucciones presentan vacíos en la mayoría de las revistas; se incumple con la regularidad de publicación, pues las revistas no tienen actualizados sus números de publicación; el alcance (scope) aún es incompleto, al igual que la ubicación geográfica de los autores y árbitros.

La difusión y visibilidad es otra dimensión a la cual se recomienda prestar atención, pues su indexación apenas alcanza Latindex, y en Publindex su clasificación es precaria, siendo esta la base de datos nacional, encargada de evaluar y categorizar las revistas en Colombia. Las dimensiones que se muestran en las siguientes tablas, dejan ver los aspectos más relevantes para que una revista sea científica, pueda mantener su indexación, o incluso pueda ser incluida en las grandes bases de datos internacionales.

La tabla 2 muestra el puntaje obtenido por las revistas en su Calidad formal, a la que se otorgan 10 puntos, distribuidos en cuatro aspectos, teniendo mayor importancia la calidad del sitio web, respecto a la cual solo tres revistas se acercan al puntaje deseado. A pesar de que los artículos tienden a tener una buena calidad, no así los sitios web, lo que denota una carencia de información en aspectos como comité científico y editorial, filiación de autores, áreas temáticas, y una página falta de dinámica, a consecuencia de lo cual la revista pierde el interés de los lectores, desconociéndose las razones por las cuales ello se presenta.

Tabla 2. Calidad Formal.

\begin{tabular}{lccccccccc}
\hline \multicolumn{1}{c}{ Calidad formal 10\% } & $\begin{array}{c}\text { Puntaje } \\
\text { Máx. }\end{array}$ & R 1 & R 2 & R 3 & R 4 & R 5 & R 6 \\
\hline $\begin{array}{l}\text { Calidad formal de los artículos } \\
\begin{array}{l}\text { Calidad del resumen y de las } \\
\text { palabras clave }\end{array}\end{array}$ & 2 & 2 & 2 & 1 & 2 & 2 & 2 \\
\hline
\end{tabular}




\begin{tabular}{lccccccccc}
\hline \multicolumn{1}{c}{ Calidad formal 10\% } & $\begin{array}{c}\text { Puntaje } \\
\text { Máx. }\end{array}$ & R 1 & R 2 & R 3 & R 4 & R 5 & R 6 \\
\hline $\begin{array}{l}\text { Filiación profesional completa de los } \\
\text { autores }\end{array}$ & 2 & 1 & 1 & 2 & 2 & 1 & 2 \\
$\begin{array}{l}\text { Calidad del sitio web } \\
\text { Subtotal calidad formal }\end{array}$ & 4 & 1 & 1 & 2 & 3 & 3 & 3 \\
\hline
\end{tabular}

Tabla 3. Calidad Informativa.

\begin{tabular}{|c|c|c|c|c|c|c|c|}
\hline Calidad informativa $10 \%$ & $\begin{array}{l}\text { Puntaje } \\
\text { Máx. }\end{array}$ & R 1 & R 2 & R 3 & R 4 & R 5 & R 6 \\
\hline Instrucciones a los autores & 4 & 1 & 1 & 3 & 4 & 1 & 3 \\
\hline Código de ética de publicación & 3 & 0 & 0 & 0 & 1 & 0 & 0 \\
\hline $\begin{array}{l}\text { Política de Open Access y Derechos } \\
\text { de Autor }\end{array}$ & 3 & 0 & 0 & 1 & 3 & 0 & 3 \\
\hline Subtotal calidad informativa & 10 & 1 & 1 & 4 & 8 & 1 & 6 \\
\hline
\end{tabular}

En la tabla 3 se observa que al criterio Calidad informativa se le otorgan 10 puntos, distribuidos en tres aspectos, teniendo mayor relevancia la instrucción a los autores, con ausencia casi total en el código de ética de publicación y mucha regularidad en la política de acceso abierto. Es necesario que exista claridad, tanto para los editores como para los investigadores, sobre los aspectos de la calidad informativa, sobre el código de ética de publicación, para saber si se adhiere o no a las normas de la revista. Así mismo, se deben dar a conocer los términos de la política de acceso abierto, de modo que el autor pueda determinar si somete, o no, su manuscrito a la revista. Se requiere de las revistas que den a conocer las licencias Creative Commons ${ }^{2}$ e informar sobre el acceso que tienen sus publicaciones.

2 Licencias basadas en el derecho de autor que definen los términos en que la obra puede ser usada, qué derechos desean entregar y en qué condiciones lo harán. 
En la tabla 4 se hace mención a la Calidad editorial, a la que se otorgan 20 puntos distribuidos en 4 aspectos, cada uno con puntaje de 5. La regularidad solo la manifiesta una revista, y los intervalos de publicación solo dos. Se puede observar que las revistas padecen de una inconstancia en la publicación de los números, de la cual solo una cumple con este parámetro, y los intervalos de publicación no se cumplen a cabalidad, dado que solo dos revistas se ajustan a ello, siendo lo ideal que todas lo hicieran. No es claro si esto obedece a falta de artículos para publicar, o a falta de capacidad editorial de la revista (tiempo, recurso humano) para gestionar la revisión y publicación de los artículos.

Tabla 4. Calidad Editorial.

\begin{tabular}{|c|c|c|c|c|c|c|c|}
\hline Calidad editorial $20 \%$ & Puntaje Máx. & R 1 & R 2 & R 3 & R 4 & R 5 & R 6 \\
\hline Scope (alcance) & 5 & 3 & 1 & 3 & 1 & 3 & 3 \\
\hline Anonimato en la revisión & 5 & 5 & 0 & 5 & 5 & 5 & 5 \\
\hline Regularidad & 5 & 0 & 0 & 5 & 0 & 0 & 0 \\
\hline $\begin{array}{l}\text { Intervalo de publicación: } \\
\text { Recepción/Aceptación/ } \\
\text { Publicación }\end{array}$ & 5 & 5 & 0 & 0 & 3 & 5 & 1 \\
\hline Subtotal calidad editorial & 20 & 13 & 1 & 13 & 9 & 13 & 9 \\
\hline
\end{tabular}

Tabla 5. Calidad Científica.

\begin{tabular}{lccccccc}
\hline \multicolumn{1}{c}{ Calidad científica 30\% } & Puntaje Máx. & R 1 & R 2 & R 3 & R 4 & R 5 & R 6 \\
\hline $\begin{array}{l}\text { Porcentaje de artículos de } \\
\text { investigación }\end{array}$ & 5 & 5 & 5 & 3 & 5 & 5 & 3 \\
$\begin{array}{l}\text { Comité Editorial (apertura } \\
\text { institucional) }\end{array}$ & 5 & 5 & 1 & 3 & 3 & 5 & 5 \\
$\begin{array}{l}\text { Comité Científico (apertura } \\
\text { geográfica) }\end{array}$ & 5 & 5 & 0 & 5 & 5 & 5 & 5 \\
$\begin{array}{l}\text { Autoría: apertura geográfica } \\
\begin{array}{l}\text { Autoría: grado de } \\
\text { endogamia institucional }\end{array}\end{array}$ & 5 & 1 & 1 & 5 & 5 & 1 & 5 \\
\end{tabular}




\begin{tabular}{lccccccc}
\hline \multicolumn{1}{c}{ Calidad científica 30\% } & Puntaje Máx. & R 1 & R 2 & R 3 & R 4 & R 5 & R 6 \\
\hline $\begin{array}{l}\text { Árbitros (apertura } \\
\text { geográfica) }\end{array}$ & 5 & 0 & 0 & 0 & 5 & 5 & 0 \\
\begin{tabular}{l} 
Subtotal calidad científica \\
\hline
\end{tabular} & 30 & 17 & 8 & 19 & 28 & 22 & 21 \\
\hline
\end{tabular}

La tabla 5 muestra el resultado de la evaluación de la Calidad científica de las revistas, a la que se asignan 30 puntos, distribuidos en 6 aspectos, cada uno con 5 puntos. Los resultados reflejan ausencia de datos sobre los árbitros, pues solo dos revistas informan al respecto. La apertura geográfica, como el grado de endogamia de las revistas, no se dan a conocer. Ambos aspectos dejan dudas sobre el proceso para garantizar una mejor calidad de los artículos y de la revista. Es necesario que exista claridad en esta dimensión, dado que es una de las más importantes para dar categoría en una revista de carácter científico-académica.

En la tabla 6 se evalúa la difusión y visibilidad de las revistas en la base de datos nacional y en bases de datos internacionales, y se observa ausencia en ellas. Es pobre su clasificación en Publindex, y sólo existe predominancia en Latindex. Es de cuestionarse por qué las revistas no han trascendido a bases de datos más rigurosas a nivel internacional, sin que se pueda establecer si obedece a falta de gestión editorial, a baja calidad de los artículos sometidos, a falta de postulación de manuscritos, o a otros motivos, lo que no permite dar a conocer ampliamente la revista.

Tabla 6. Difusión y visibilidad.

\begin{tabular}{|c|c|c|c|c|c|c|c|}
\hline Difusión y visibilidad 30\% & Puntaje Máx. & R 1 & R 2 & R 3 & R 4 & R 5 & R 6 \\
\hline $\begin{array}{l}\text { Indización en bases de datos } \\
\text { de citaciones internacionales } \\
\text { (Web of Science, Scopus) }\end{array}$ & 12 & 0 & 0 & 0 & 0 & 0 & 0 \\
\hline $\begin{array}{l}\text { Indización en bases de datos } \\
\text { multidisciplinares regionales } \\
\text { (SciELO Colombia; SciELO } \\
\text { Citation Index; Redalyc) }\end{array}$ & 10 & 0 & 0 & 0 & 0 & 0 & 0 \\
\hline
\end{tabular}




\begin{tabular}{lccccccc}
\hline Difusión y visibilidad 30\% & Puntaje Máx. & R 1 & R 2 & R 3 & R 4 & R 5 & R 6 \\
\hline $\begin{array}{l}\text { Características Latindex (36 } \\
\text { características) }\end{array}$ & 4 & 4 & 4 & 0 & 4 & 4 & 4 \\
$\begin{array}{l}\text { Clasificación Publindex 2014-I } \\
\begin{array}{l}\text { Subtotal calidad difusión y } \\
\text { visibilidad }\end{array}\end{array}$ & 4 & 1 & 1 & 0 & 2 & 1 & 2 \\
\hline
\end{tabular}

Tabla 7. Calificación de evaluación de las revistas.

\begin{tabular}{lccccccc}
\hline & Puntaje Máx. & R 1 & R 2 & R 3 & R 4 & R 5 & R 6 \\
\hline Calidad Formal & $10 \%$ & 6 & 6 & 7 & 9 & 8 & 9 \\
Calidad Informativa & $10 \%$ & 1 & 1 & 4 & 8 & 1 & 6 \\
Calidad Editorial & $20 \%$ & 13 & 1 & 13 & 9 & 13 & 9 \\
Calidad Científica & $30 \%$ & 17 & 8 & 19 & 28 & 22 & 21 \\
Difusión y visibilidad & $30 \%$ & 5 & 5 & 0 & 6 & 5 & 6 \\
Total Evaluación & $100 \%$ & $42 \%$ & $21 \%$ & $43 \%$ & $60 \%$ & $49 \%$ & $51 \%$ \\
\hline
\end{tabular}

En la tabla 7 se muestra el $100 \%$ de los resultados, distribuidos en los cinco aspectos evaluados (calidad formal, informativa, editorial, científica y difusión y visibilidad), donde el porcentaje más alto es $60 \%$, y el más bajo $21 \%$.

Otra dimensión que se tuvo en cuenta fue la administrativa. Este componente de la matriz elaborada por Molina et. al. (2016) se envió a los editores de las revistas seleccionadas, y solo se obtuvo respuesta de 3 de ellas, un $50 \%$ de la muestra. A continuación, como lo exponen Molina et. al. (2016), se analizan las categorías: equipo editorial, ingresos, costos, estrategias de mercadeo, manuales e instrumentos, informes estadísticos y documentos administrativos.

\section{Equipo Editorial}

En la tabla 8 se observan los diferentes roles establecidos en el equipo editorial, desde el director de la revista, hasta los editores de sección. 
Tabla 8. Cargos dentro del equipo editorial.

Adaptado de Molina et al. (2016, p.102).

\begin{tabular}{clllcc}
\hline Director & Editor & \multicolumn{1}{c}{$\begin{array}{c}\text { Director/ } \\
\text { Editor }\end{array}$} & $\begin{array}{c}\text { Asistente } \\
\text { de Edición }\end{array}$ & $\begin{array}{c}\text { Editor de } \\
\text { sección }\end{array}$ & $\begin{array}{c}\text { Horas } \\
\text { Total }\end{array}$ \\
\hline 4 h. vinculado & $\begin{array}{l}4 \mathrm{~h} \text {. Catedra, } \\
\text { pensionado }\end{array}$ & & 8 \\
& $\begin{array}{l}20 \mathrm{~h} . \\
\text { Vinculado } \\
\text { Tiempo } \\
\text { Completo }\end{array}$ & $\begin{array}{l}40 \mathrm{~h} . \\
\text { Contratista }\end{array}$ & $4 \mathrm{~h}$. Vinculado & 64 \\
& & & \\
\hline 8 h. vinculado & & & 8
\end{tabular}

Las tres revistas que aportaron datos administrativos para su evaluación, dan cuenta de que falta personal para los cargos de director, asistente de edición y editor de sección. Solo una revista cumple con los dos últimos cargos. Y la disponibilidad de horas semanales se debe cuestionar, dado que la revista que más horas tiene a disposición son 64 , una de ellas no muestra cargos ni horas, y la tercera revista solo tiene 8 horas semanales.

\section{Equipo de Apoyo}

Está compuesto por las personas que ayudan en la revista en aspectos de traducción, corrección, impresión, entre otros, como se aprecia en la tabla 9.

Tabla 9. Apoyo Editorial. Adaptado de Molina et al. (2016).

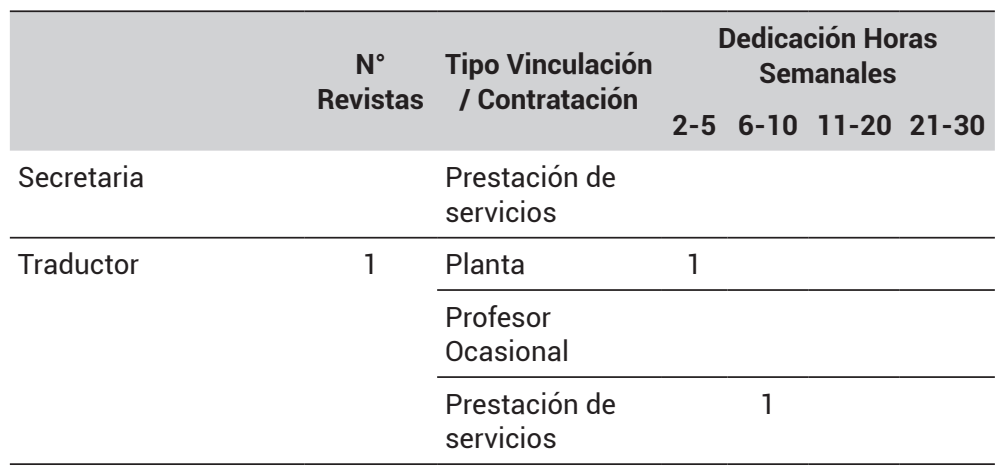




\begin{tabular}{|c|c|c|c|c|c|c|}
\hline & \multirow{2}{*}{$\begin{array}{c}\mathrm{N}^{\circ} \\
\text { Revistas }\end{array}$} & \multirow{2}{*}{$\begin{array}{l}\text { Tipo Vinculación } \\
\text { / Contratación }\end{array}$} & \multicolumn{4}{|c|}{$\begin{array}{l}\text { Dedicación Horas } \\
\text { Semanales }\end{array}$} \\
\hline & & & $2-5$ & $6-10$ & $11-20$ & $21-30$ \\
\hline \multirow[t]{2}{*}{ Corrector de Texto } & 2 & Contrato & & 1 & & \\
\hline & & $\begin{array}{l}\text { Prestación de } \\
\text { servicios }\end{array}$ & & & & 1 \\
\hline \multicolumn{7}{|l|}{ Ilustrador } \\
\hline Diagramador & & $\begin{array}{l}\text { Prestación de } \\
\text { servicios }\end{array}$ & & & & \\
\hline Impresor & & $\begin{array}{l}\text { Prestación de } \\
\text { servicios }\end{array}$ & & & & \\
\hline $\begin{array}{l}\text { Gestión de referencias } \\
\text { bibliográficas y } \\
\text { Sistema de citaciones }\end{array}$ & & $\begin{array}{l}\text { Prestación de } \\
\text { servicios } \\
\text { (Asistente) }\end{array}$ & & & & \\
\hline $\begin{array}{l}\text { Responsable del } \\
\text { Proceso Open Journal } \\
\text { System }\end{array}$ & 1 & Monitor & 1 & & & \\
\hline \multicolumn{7}{|l|}{$\begin{array}{l}\text { Responsable del } \\
\text { Proceso SciELO }\end{array}$} \\
\hline \multicolumn{7}{|l|}{$\begin{array}{l}\text { Responsable } \\
\text { de los procesos } \\
\text { con servicios de } \\
\text { indización y resumen }\end{array}$} \\
\hline $\begin{array}{l}\text { Auxiliares } \\
\text { Administrativos }\end{array}$ & 1 & Estudiante & & & 2 & \\
\hline $\begin{array}{l}\text { Auxiliares de } \\
\text { Programación }\end{array}$ & & Estudiante & & & & \\
\hline Otros ¿Cuáles? & & & & & & \\
\hline
\end{tabular}

El apoyo que tienen las revistas con personal externo a los del equipo editorial también requiere atención. Entre el equipo de apoyo están los traductores, correctores de estilo, auxiliares administrativos y responsables de la plataforma Open Journal System. 


\section{Ingresos}

La tabla 10 muestra los diferentes ingresos que obtiene la revista, bien sea por la propia revista o por fuentes externas.

Tabla 10. Ingresos de la revista. Adaptado de Molina et al. (2016).

Ingresos generados por la revista

Pagos de autores por publicar

Pagos extras por publicar ilustraciones a color,

fotografías o páginas adicionales

Publicidad

Patrocinio

Distribuidores

(Bases de datos, agregadores de contenido, etc.)

Suscripciones

Otros ¿Cuáles?

Ingresos gestionados ante fuentes distintas a la revista Internos (propios de la Universidad de Antioquia)

Centro de costo especial de la unidad editora

Fondo financiación de revistas indexadas

Fondo financiación de revistas especializadas

Otros ¿Cuáles?

Externos (distintos de la Universidad de Antioquia)

Subvenciones

Donaciones

Convenios de cooperación

Otros. ¿Cuáles?
$\mathbf{N}^{\circ}$ Revistas

Los ingresos que perciben las revistas son casi nulos. Solo una revista recibe ingresos, y solo por el Fondo financiación de revistas indexadas. Las revistas no reciben ingresos por publicaciones, 
ni suscripciones, dada la política de acceso libre. Si se compara con los costos que tienen para sistema de publicación digital, procesamiento de manuscritos, edición e ilustración, recursos humanos, mercado y promoción, estos no se alcanzan a cubrir.

\section{Costos}

Tabla 11. Costos de la revista. Adaptado de Molina et al. (2016).

\begin{tabular}{lc}
\hline \multicolumn{1}{c}{ Costos } & N $^{\circ}$ revistas \\
\hline $\begin{array}{l}\text { Sistema de publicación digital (incluido desarrollo técnico y } \\
\text { diseño del sitio web) }\end{array}$ & 1 \\
\hline Sistema de gestión editorial online de los manuscritos & 1 \\
\hline Formateado de contenidos & 2 \\
\hline Hospedaje de la Web y archivo de los artículos & \\
\hline $\begin{array}{l}\text { Primera copia, incluye actividades tales como: procesamiento } \\
\text { de manuscritos, aceptación inicial, identificación de árbitros, } \\
\text { procesamiento de evaluaciones, procesamiento de manuscritos }\end{array}$ & \\
$\begin{array}{l}\text { aceptados para publicación, y lo relacionado con edición, } \\
\text { formateo, ilustración, diseño gráfico, indización, codificación, }\end{array}$ & 2 \\
revisión de pruebas, composición final & 1 \\
\hline $\begin{array}{l}\text { Recursos Humanos } \\
\text { Administración, incluye registro y nómina de personal, } \\
\text { contabilidad, mantenimiento y administración de equipos, } \\
\text { manejo de inventarios, asignación de espacios, administración } \\
\text { de pagos de regalías y derechos de autor }\end{array}$ & \\
\hline $\begin{array}{l}\text { Mercadeo y Promoción } \\
\text { Imprenta }\end{array}$ & 2 \\
\hline $\begin{array}{l}\text { Distribución, incluye costos de envío de ejemplares físicos } \\
\text { por correo urbano, nacional e internacional, o costos de } \\
\text { intermediación de distribuidores tales como librerías, ferias y } \\
\text { similares }\end{array}$ & 1 \\
\hline
\end{tabular}

La tabla 11 muestra los costos que tienen las revistas, según el apartado que consideran más relevante, entre los cuales están desarrollo de páginas, hospedaje de la Web, imprenta, mercado, etc. 


\section{Estrategia de mercado}

En la tabla 12 se muestran las diferentes estrategias para generar mercado y divulgar las revistas por diferentes medios electrónicos.

Tabla 12. Estrategia de mercado. Adaptado de Molina et al. (2016).

\section{Estrategia}

Eventos académicos y de extensión, con o sin stand. Incluye los organizados por la propia unidad académica editora y modalidades de promoción y venta

Ferias del libro, por medio de la editorial de la universidad Distribución de ejemplares por cortesía o donación Promociones en acontecimientos especiales de la universidad

Presentación de la revista en programas de inducción Indexación de la revista en las bases de datos más prestigiosas

Consignación de la revista en la librería de la universidad

Promoción del formato en papel, en el mismo sitio web de la revista

Editores y distribuidores externos

Alianzas con servicios de bases de datos (EBSCO)

Invitación anual a renovar suscripciones

Ninguna estrategia, por falta de tiempo

Medios de divulgación

Redes sociales

Indización en bases de datos

Correo electrónico

Listas de distribución

Sitio web de la revista

Sitio web de la unidad académica

Sitio web en inglés

Bases de datos de la unidad académica
$\mathbf{N}^{\circ}$ ocurrencias 
Las estrategias de mercadeo y divulgación solo están en redes sociales, correo electrónico y sitio web de la revista. Solo una hace divulgación y mercadeo en eventos académicos y de extensión.

\section{Manuales e instrumentos}

Tabla 13. Manual e instrumentos. Adaptado de Molina et al. (2016).

\begin{tabular}{|c|c|c|}
\hline Manual de Estilo & \multicolumn{2}{|c|}{ Normas APA sexta edición } \\
\hline $\begin{array}{l}\text { Sistema de referencias bibliográficas y } \\
\text { citaciones }\end{array}$ & & \\
\hline \multicolumn{3}{|l|}{ Normas para la elaboración de resúmenes } \\
\hline $\begin{array}{l}\text { Tesauros para la selección de } \\
\text { descriptores }\end{array}$ & Alfabético & $\begin{array}{l}\text { DESC de LILACS } \\
\text { Bireme }\end{array}$ \\
\hline $\begin{array}{l}\text { Fuentes utilizadas para la elaboración de } \\
\text { índices }\end{array}$ & \multicolumn{2}{|c|}{ Metodología de la investigación } \\
\hline Software para la detección de plagio & my.plag.es & Cross Check \\
\hline $\begin{array}{l}\text { Software para la gestión de referencias } \\
\text { bibliográficas }\end{array}$ & Citavi & \\
\hline $\begin{array}{l}\text { Otros. ¿Cuáles? } \\
\text { (Agregue las filas que considere } \\
\text { necesarias) }\end{array}$ & & \\
\hline
\end{tabular}

Los manuales e instrumentos indican a los autores y evaluadores qué normas se han de cumplir en los artículos, qué medios se han de usar para detectar fraudes, referencias, entre otros.

\section{Informes estadísticos}

Los datos registrados en la tabla 14 recogen los trabajos recibidos, aprobados y publicados en las revistas entre el inicio de los años 2016 y 2018. Respectivamente se anexan los porcentajes de trabajos aprobados, publicados / aprobados, publicados / recibidos y la tasa de rechazo. 
Tabla 14. Informes estadísticos. Adaptado de Molina et al. (2016).

\begin{tabular}{llll}
\hline Trabajos recibidos & 88 & 90 & 80 \\
\hline Trabajos aprobados & 73 & 28 & 40 \\
\hline$\%$ trabajos aprobados & 83 & 31 & 50 \\
\hline Tasa de rechazo & 17 & 68 & 50 \\
\hline Trabajos publicados & 72 & 20 & 40 \\
\hline \% trabajos publicados/ aprobados & 99 & 71 & 100 \\
\hline$\%$ trabajos publicados/ recibidos & 82 & 22 & 50 \\
\hline
\end{tabular}

En el informe estadístico que se obtuvo por las tres revistas, los artículos recibidos entre el primer semestre de 2016 y el primer semestre de 2018, estaban entre 80 y 90. La tasa de rechazo refleja una gran demanda y exigencia de las revistas; entre más alta la tasa de rechazo, mejor se considera la revista (Molina et al., 2016). La más baja fue de 17 y la más alta de 68 .

\section{Documentos administrativos}

En la tabla 15 se hace mención de los diferentes documentos administrativos, como plan de acción, formatos, informes, código de ética, entre otros.

Tabla 15. Documentos administrativos. Tomado de Molina et al. (2016).

\begin{tabular}{lccc}
\hline Reglamento Interno & No & Sí & Sí \\
\hline Formato para el Arbitraje de Artículos & Sí & Sí & Sí \\
\hline Formatos acuerdos de propiedad intelectual & & Sí & Sí \\
\hline Código de ética & & Sí & Sí \\
\hline Plan de Acción & & Sí & Sí \\
\hline Informes Estadísticos & No & No & No \\
\hline Registro de suscriptores e instituciones para canje & No & Sí & Sí \\
\hline
\end{tabular}




\section{DISCUSIÓN}

A partir de los resultados del estudio, se puede inferir que las revistas de Educación Física y áreas afines se encuentran en una fase que exige mejoras significativas, destacando los siguientes aspectos:

\section{Calidad formal}

Las revistas poseen un buen aspecto en cuanto a la calidad formal de los artículos y palabras clave. Sin embargo, es importante mejorar la calidad del sitio web, para que sea más dinámica y permanezca actualizada su información.

\section{Calidad informativa}

En las revistas seleccionadas no se encuentran documentos sobre código de ética, política de Open Access y derechos de autor. Las instrucciones a los autores son el único ítem que se ajusta un poco a criterios establecidos. En general, las revistas tienen un gran trabajo pendiente en este aspecto de calidad informativa, pues los autores necesitan información e instrucciones para la publicación.

\section{Calidad editorial}

Las revistas presentan una notable deficiencia en este ítem, que, como lo expresan Molina et al. (2016), "es la carta de presentación de la revista ante autores, árbitros y lectores". Por ello se recomienda a las revistas prestar atención en este aspecto.

Otra notable falencia es el intervalo de Publicación/Aceptación/Publicación, que solo una revista cumple cabalmente.

\section{Calidad científica}

De las seis revistas, solo una alcanza a cumplir los criterios de calidad científica. Se observa un mayor vacío en la apertura geo- 
gráfica de los árbitros, dado que solo dos revistas brindan información en sus publicaciones sobre el origen de los evaluadores.

\section{Difusión y visibilidad}

Este representa el mayor reto en el cual las revistas de Educación Física deben trabajar, pues ninguna revista aparece en indexadores internacionales, excepto en Latindex, y su clasificación en Publindex no es mayor a B., lo que refleja la baja difusión y visibilidad que tienen estas revistas.

De manera global, y en comparación con el estudio de Molina et al., (2016) se destaca lo siguiente:

Molina et al. reportan en su investigación que sólo una revista cumple con el ítem de alcance (scope), la mayoría de las revistas están ausentes de las bases de datos internacionales, y paulatinamente aumentan las revistas cuando los indexadores son menos rigurosos para su inclusión en ellos, al punto de ser escasas las revistas sin indexador. En comparación con las revistas del área de Educación Física en Colombia evaluadas, los resultados son similares, dando a entender que la problemática no es de una sola revista, sino de las universidades en general, que tienen la función de producir conocimiento y darlo a conocer por medio de las publicaciones. En este sentido, su progreso es muy paulatino, y, como lo señala Urrego (2017), "los problemas de edición, difusión y divulgación de la producción de conocimiento en Educación Física, Deporte y Recreación en Colombia, son crónicos y críticos".

\section{REFERENCIAS}

1. Corera, E., \& Molina, S. (2016). La edición universitaria de revistas científicas. Revista Interamericana de Bibliotecología, 39(3), 277-288. doi: 10.17533/udea.rib.v39n3a05 
2. Day, R. (2005). Cómo escribir y publicar trabajos científicos (3aㅡ. Ed.). Organización Panamericana de la Salud. doi: 10.1590/ S0864-34662008000300018

3. Molina, M., Gómez, J., Corera, E., Vélez, M., Mejía, K., \& Quiróz, P. (2016). La edición de revistas científicas en la Universidad de Antioquia. Informe ejecutivo. Universidad de Antioquia. Medellín. Disponible en http://bibliotecadigital.udea.edu.co/dspace/handle/10495/8805

4. Osca, J., Haba, J., Minguez, O., Navarro, G., Velasco, E., \& Salom, L. (2008). Difusión y factor de impacto nacional e internacional de las revistas científicas españolas. Anales de Documentación, 11, 145-164. doi: 10.6018/analesdoc.11.0.24861

5. Rodríguez, Y., Rubio, Y., \& Solórzano, E. (2007). Las ciencias sociales en Cuba: una mirada desde una perspectiva métrica. ACIMED, 16(6). Disponible en http://eprints.rclis.org/11019/

6. Rozemblum, C., Unzurrunzaga, C., Banzato, G., \& Pucacco, C. (2015). Calidad editorial y calidad científica en los parámetros para inclusión de revistas científicas en bases de datos en Acceso Abierto y comerciales. Palabra Clave, 4(2), 64-80. Disponible en http://www.palabraclave.fahce.unlp.edu.ar/article/view/PCv4n2a01/6596

7. Urrego, L. (2017). Problemas de edición de la producción de conocimiento en Educación Física (Ensayo inédito). Universidad de Antioquia. 